\title{
ADEQUAÇÃO DOS MODELOS DE LANGMUIR E FREUNDLICH NA ADSORÇÃO DE COBRE EM SOLO ARGILOSO DO SUL DO BRASIL
}

\author{
M. S. Gonçalves ${ }^{1}$; J. P. Bettin ${ }^{2}$; L. C. S. Silva Junior ${ }^{2}$; S. C. Sampaio ${ }^{3}$ e T. C. Dal Bosco ${ }^{4}$ \\ ${ }^{1}$ Universidade Tecnológica Federal do Paraná (UTFPR) \\ ${ }^{2}$ Universidade Estadual do Oeste do Paraná (UNIOESTE) \\ morgana@utfpr.edu.br
}

Artigo submetido em maio/2013 e aceito em agosto/2013

\section{RESUMO}

Os estudos da capacidade de adsorção de metais pesados em solos podem ser empregados para avaliar a contribuição dos mesmos na contaminação de águas superficiais e subterrâneas. O propósito deste trabalho foi estudar a adequação dos modelos de Langmuir e de Freundlich para descrever a adsorção de cobre em latossolo vermelho distrófico úmbrico de textura argilosa. Para determinação da capacidade de adsorção de cobre no solo, utilizou-se 2,000 g de solo adicionados de $10 \mathrm{~mL}$ de $\mathrm{CaCl}_{2}$ 0,01 mol L ${ }^{-1}$ e $10 \mathrm{~mL}$ de soluções de $\mathrm{CuCl}_{2}$ em diferentes concentrações. A capacidade máxima de adsorção de cobre no solo, determinada pelo modelo de Langmuir, foi de $2,95 \mathrm{mg} \mathrm{g}^{-1}$. Além disso, os resultados demonstraram que o modelo de Freundlich possui melhor ajuste aos dados experimentais, simulando adequadamente a adsorção do cobre no solo em estudo.

PALAVRAS-CHAVE: adsorção, metais pesados, isotermas, latossolo

\section{LANGMUIR AND FREUNDLICH MODELS ADEQUACY IN THE COPPER ADSORPTION IN CLAY SOIL OF SOUTHERN BRAZIL}

\begin{abstract}
Studies of the adsorption of heavy metals in soil can be used to assess this contribution in the contamination of surface and groundwater. This work had as objective to study the adequacy of the models of Langmuir and Freundlich to describe the adsorption of copper in a Rhodic Hapludox soil with clay texture. To determine the adsorption capacity of copper in the soil, we used 2.000 $\mathrm{g}$ of soil added $10 \mathrm{~mL}$ of $\mathrm{CaCl}_{2} 0.01 \mathrm{~mol} \mathrm{~L}^{-1}$ and $10 \mathrm{~mL}$
\end{abstract}

solutions of $\mathrm{CuCl}_{2}$ with different concentrations. The maximum adsorption capacity of copper in the soil, determined by the Langmuir model, was $2.95 \mathrm{mg} \mathrm{g}^{-1}$. Moreover, the results showed that the Freundlich model showed better fit to the experimental data, adequately simulate the adsorption of copper in the soil under study. 


\section{ADEQUAÇÃO DOS MODELOS DE LANGMUIR E FREUNDLICH NA ADSORÇÃO DE COBRE EM SOLO ARGILOSO DO SUL DO BRASIL}

\section{INTRODUÇÃO}

As concentrações da maioria dos metais pesados, encontrados nos compartimentos ambientais, são normalmente pequenas e não causam problemas diretos à saúde. Entretanto, quando ocorrem intervenções no ambiente como contaminações de solos e recursos hídricos, algumas substâncias sofrem biomagnificação, ou seja, suas concentrações aumentam progressivamente ao longo de uma cadeia alimentar ecológica, de tal forma que os predadores possuem maiores concentrações (PIVELI E KATO, 2006).

Os metais pesados diferenciam-se dos compostos orgânicos tóxicos, por serem nãodegradáveis, de maneira que podem acumular-se no ambiente, no qual manifestam sua toxicidade, fixando-se de forma final nos solos e sedimentos (BAIRD, 2004).

O cobre é um metal usado amplamente nas áreas industrial e agrícola, de forma que pode ser acrescido ao meio a partir da disposição de resíduos sólidos industriais ou domésticos, uso de agroquímicos e aplicação de dejetos animais e efluentes ao solo. Vasconcelos et al. (1997) citam que em grandes concentrações no solo, o cobre pode proporcionar caráter tóxico aos vegetais.

Cada tipo de solo, de acordo com suas características, possui uma determinada capacidade de retenção de metais pesados. Quando se excede esta capacidade, o metal pode sofrer lixiviação ou ser carreado pela chuva e pode atingir águas superficiais e subterrâneas. Assim, a determinação da capacidade de retenção de metais contaminantes no solo é de fundamental importância quando relacionada à solubilidade e à disponibilidade do poluente no meio.

Estudos da capacidade máxima de adsorção de metais pesados em um solo podem ser empregados para avaliar a contribuição destes na contaminação de corpos hídricos. A matéria orgânica existente no solo e a quantidade de argila são os principais atributos responsáveis pela fixação dos poluentes devido à alta complexidade de sua estrutura. Entretanto, $\mathrm{O} \mathrm{pH}$ e a capacidade de troca iônica também interferem na adsorção de metais em solos (SOUZA, 2006).

Uma das maneiras de estudar a retenção de metais pesados no solo inicia-se com o uso de isotermas de adsorção (BÖTTCHER, 1997; HINZ, 2001). Dentre os modelos de isotermas existentes que descrevem a adsorção de metais no solo, os mais utilizados são os propostos por Langmuir e Freundlich. De acordo com Nascimento e Fontes (2004) essas equações, embora não esclareçam sobre os mecanismos químicos associados à adsorção, são facilmente aplicáveis para adsorção de quaisquer íons pelo solo com a vantagem de fornecer informações que possam ser relacionadas às propriedades dos solos.

\section{MATERIAL E MÉTODOS}

O solo utilizado no experimento, de textura argilosa e classificado como latossolo vermelho distrófico úmbrico (EMBRAPA, 2006), foi coletado no município de Francisco Beltrão, 
PR, cuja amostra foi obtida a uma profundidade entre 0 e $30 \mathrm{~cm}$. $O$ solo teve restos de raízes removidas manualmente, após secagem foi desagregado e peneirado em uma peneira com 2,0 $\mathrm{mm}$ de abertura da tela. Realizou-se a determinação das características físico-químicas do solo (Tabela 1), de acordo com metodologia descrita por EMBRAPA (1997) e Tedesco et al. (1995).

Tabela 1 - Caracterização físico-química do solo.

\begin{tabular}{ccc}
\hline \multicolumn{3}{c}{ Composição Granulométrica } \\
\hline Parâmetro & Unidade & Valor \\
\hline Areia & $\%$ & 10,00 \\
Silte & $\%$ & 20,00 \\
Argila & Características Químicas & 70,00 \\
\hline \multicolumn{3}{c}{ Unidade } \\
\hline Parâmetro & - & Valor \\
\hline $\mathrm{Ca}\left(\mathrm{CaCl}_{2}\right)$ & $\mathrm{cmol}_{\mathrm{c}} \mathrm{dm}^{-3}$ & 5,10 \\
$\mathrm{Mg}$ & $\mathrm{cmol}_{\mathrm{c}} \mathrm{dm}^{-3}$ & 5,17 \\
$\mathrm{~K}$ & $\mathrm{cmol}_{\mathrm{c}} \mathrm{dm}^{-3}$ & 3,53 \\
$\mathrm{Al}$ & $\mathrm{cmol}_{\mathrm{c}} \mathrm{dm}^{-3}$ & 0,25 \\
$(\mathrm{H}+\mathrm{Al})$ & $\mathrm{cmol}_{\mathrm{c}} \mathrm{dm}^{-3}$ & 0,00 \\
$\mathrm{~S}$ & $\mathrm{cmol}_{\mathrm{c}} \mathrm{dm}^{-3}$ & 5,76 \\
$\mathrm{CTC}$ & $\mathrm{cmol}_{\mathrm{c}} \mathrm{dm}^{-3}$ & 8,95 \\
$\mathrm{C}$ & $\mathrm{g} \mathrm{dm}^{-3}$ & 14,71 \\
$\mathrm{MO}$ & $\mathrm{g} \mathrm{dm}^{-3}$ & 20,74 \\
$\mathrm{P}$ & $\mathrm{mg} \mathrm{dm}^{-3}$ & 35,67 \\
$\mathrm{Fe}$ & $\mathrm{mg} \mathrm{dm}^{-3}$ & 5,70 \\
$\mathrm{Mn}$ & $\mathrm{mg} \mathrm{dm}^{-3}$ & 21,69 \\
$\mathrm{Cu}$ & $\mathrm{mg} \mathrm{dm}^{-3}$ & 46,67 \\
$\mathrm{Zn}$ & $\mathrm{mg} \mathrm{dm}^{-3}$ & 7,84 \\
$\mathrm{~V}$ & $\%{ }^{2}$ & 63,00 \\
& & 60,84 \\
\hline
\end{tabular}

No experimento de adsorção, foram pesados dois gramas de solo e misturados com $10 \mathrm{~mL}$ de solução de $\mathrm{CaCl}_{2} 0,01 \mathrm{~mol} \mathrm{~L}^{-1}, 10 \mathrm{~mL}$ de água destilada e $10 \mathrm{~mL}$ de solução de $\mathrm{Cu}$ na forma de $\mathrm{CuCl}_{2} \cdot 2 \mathrm{H}_{2} \mathrm{O}$ nas concentrações de 13, 32, 60, 115, 117 e $286 \mathrm{mg} \mathrm{L}^{-1}$. O experimento foi realizado em triplicata e em pH natural do solo. As amostras foram agitadas a $200 \mathrm{rpm}$ e $25^{\circ} \mathrm{C}$, durante 24 h e centrifugadas a $3000 \mathrm{rpm}$ por $10 \mathrm{~min}$. O sobrenadante resultante da centrifugação foi coletado e procedeu-se a digestão das amostras com $\mathrm{HNO}_{3}$ e $\mathrm{H}_{2} \mathrm{SO}_{4}$ conforme metodologia de APHA, AWWA, WEF (1998), sendo realizada a determinação de $\mathrm{Cu}$ por espectrofotometria de absorção atômica.

A quantidade de cobre adsorvida no solo foi calculada com base na diferença de concentração na solução antes e após o experimento de adsorção, de acordo com o balanço de massa representado na Equação 1.

$q_{e}=\frac{\left(C_{0}-C_{e}\right) V}{m}$

em que:

$\mathrm{q}_{\mathrm{e}}$ - concentração de metal sorvido $\left(\mathrm{mg} \mathrm{g}^{-1}\right)$; 
$\mathrm{C}_{0}$ - concentração inicial do metal $\left(\mathrm{mg} \mathrm{L}^{-1}\right)$;

$\mathrm{C}_{\mathrm{e}}$ - concentração de equilíbrio no tempo $\mathrm{t}\left(\mathrm{mg} \mathrm{L}^{-1}\right)$;

$\mathrm{V}$ - volume da solução (L);

$\mathrm{M}$ - massa de sorvente (g).

Os resultados experimentais foram ajustados aos modelos de Langmuir (Equação 2) e de Freundlich (Equação 3).

$$
\begin{aligned}
& q_{e}=\frac{q_{0} K_{L} C_{e}}{1+K_{L} C_{e}} \\
& q_{e}=K_{f} C_{e}^{1 / n}
\end{aligned}
$$

em que:

$\mathrm{q}_{0}$ - capacidade máxima de adsorção $\left(\mathrm{mg} \mathrm{g}^{-1}\right)$;

$\mathrm{K}_{\mathrm{L}}$ - constante relacionada à energia de ligação do metal no solo $\left(\mathrm{mg} \mathrm{L}^{-1}\right)$;

$\mathrm{K}_{\mathrm{f}}$ - constante de Freundlich $\left(\mathrm{mg} \mathrm{g}^{-1}\right)$;

$\mathrm{n}$ - parâmetro de afinidade do solo pelo soluto (adimensional).

A significância estatística dos coeficientes de determinação $\left(R^{2}\right)$, encontrados para os modelos de Langmuir e Freundlich, foi testada ao grau de 5\% de significância.

\section{RESULTADOS E DISCUSSÃO}

Com base nos dados experimentais da concentração de cobre após o equilíbrio $\left(C_{e}\right)$, calculou-se a quantidade do metal adsorvido no solo. Os resultados obtidos estão expressos na Figura 1. Observou-se que nas menores concentrações de cobre adicionadas ao solo, a adsorção ocorreu com maior intensidade, caracterizando a afinidade entre o metal e os constituintes do solo. Conforme houve o aumento das concentrações do metal inicialmente aplicado, ocorreu o aumento da concentração do mesmo na solução de equilíbrio, demonstrando a sequente diminuição da adsorção e indicando a saturação dos sítios sortivos existentes no solo.

O solo em estudo possui valores elevados de argila, matéria orgânica e CTC que favorecem a adsorção do cobre e dificultam sua mobilidade no ambiente. Sodré et al. (1999) observaram que solos com maiores valores de CTC demonstraram alta correlação positiva entre os sítios de retenção dos cátions e de moléculas orgânicas com os sítios de adsorção de cobre.

Outro atributo do solo que influenciou na adsorção do cobre foi o pH (Tabela 1). Linhares et al. (2008) citam que, em experimento realizado pelos autores, os solos com maior afinidade pelos metais cádmio e chumbo, apesar de diferirem entre si em algumas propriedades, possuíam em comum valores de $\mathrm{pH}$ maiores que aqueles correspondentes aos solos como menor afinidade pelos elementos ( $\mathrm{pH}$ em torno de 4,3). Segundo Garcia-Miragaya e Page (1978) solos mais ácidos possuem tendência a liberar mais íons $\mathrm{H}^{+}$para a solução, que competem com os metais pelos sítios de adsorção na superfície adsorvente do solo. 


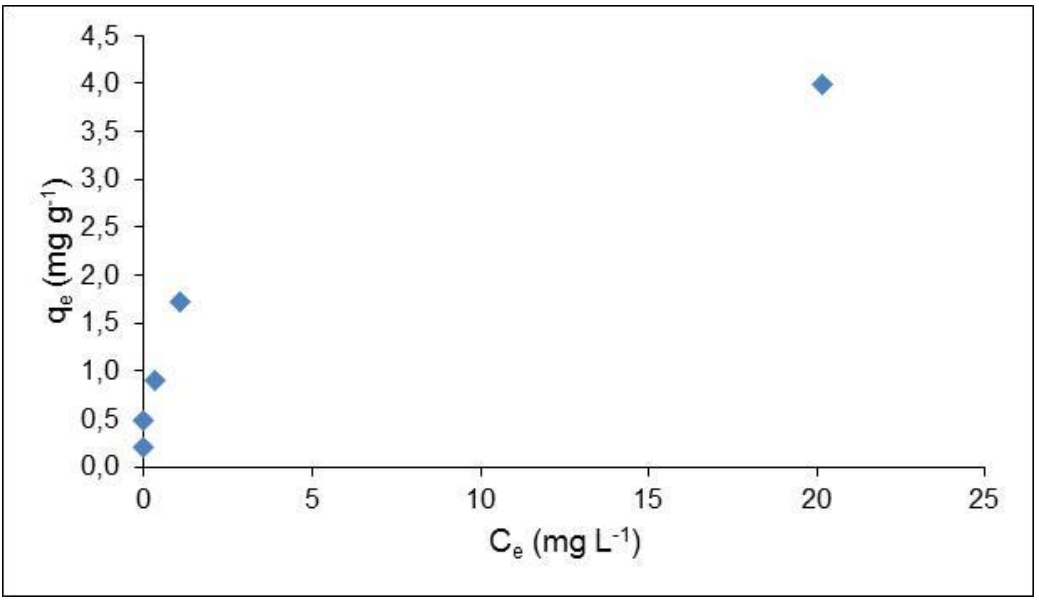

Figura 1 - Curva de adsorção de Cu de acordo com a concentração na solução de equilíbrio.

Os coeficientes de determinação obtidos pelos modelos de Langmuir e Freundlich (Tabela 2) foram significativos ao nível de $5 \%$ de significância $(P<0,05)$, entretanto, o modelo de Freundlich oferece maior coeficiente de determinação $\left(R^{2}=0,962\right)$ descrevendo mais adequadamente o processo de adsorção de cobre no solo. De acordo com Arias et al .(2006), o modelo de Freunclich pode ser utilizado para demonstrar a adsorção sobre sólidos com superfície heterogênea, mostrando-se frequentemente superior ao modelo de Langmuir para adsorção de cátions e ânions nos solos.

Tabela 2 - Equações de regressão, coeficientes de determinação e valor $\mathbf{P}$ das isotermas linearizadas de Langmuir e Freundlich.

\begin{tabular}{lccc}
\hline \multicolumn{1}{c}{ Modelo } & Equação & $\mathrm{R}^{2}$ & $\mathrm{P}$ \\
\hline Langmuir & $\frac{1}{\mathrm{q}_{\mathrm{e}}}=0,339+0,277 \frac{1}{\mathrm{C}_{\mathrm{e}}}$ & 0,933 & $0,034^{*}$ \\
Freundlich & $\log q_{e}=0,351+0,145 \log C_{e}$ & 0,962 & $0,019^{*}$ \\
\hline
\end{tabular}

* Significativo a 5\% de significância.

De acordo com os parâmetros relacionados na Tabela 3 para a isoterma de Langmuir, observa-se elevado valor da capacidade máxima de adsorção $\left(2,95 \mathrm{mg} \mathrm{g}^{-1}\right)$, com alta energia de ligação do metal no solo $\left(\mathrm{K}_{\mathrm{L}}\right)$, corroborando com resultados obtidos por Nascimento e Fontes (2004), que encontraram valores de $1,70 \mathrm{mg} \mathrm{g}^{-1}$ para a capacidade máxima de adsorção de cobre em latossolo vermelho de textura argilosa, e 1,49 $\mathrm{mg} \mathrm{L}^{-1}$ para a energia de ligação do metal no solo. O valor da energia de ligação demonstra que a maior parte dos sítios de adsorção é de alta afinidade pelo elemento, o que aumenta sua fixação no solo, reduz sua movimentação e redisponibilização para o ambiente, com importância significativa em estudos ambientais (LINHARES et al., 2009).

Tabela 3 - Parâmetros das isotermas de adsorção de Langmuir e Freundlich.

\begin{tabular}{cccc}
\hline \multicolumn{2}{c}{ Langmuir } & \multicolumn{2}{c}{ Freundlich } \\
\hline $\mathrm{q}_{0}\left(\mathrm{mg} \mathrm{g}^{-1}\right)$ & $\mathrm{K}_{\mathrm{L}}\left(\mathrm{mg} \mathrm{L}^{-1}\right)$ & $\mathrm{K}_{\mathrm{f}}\left(\mathrm{mg} \mathrm{g}^{-1}\right)$ & $1 / \mathrm{n}$ \\
\hline 2,95 & 1,22 & 1,40 & 0,35 \\
\hline
\end{tabular}


Quanto aos parâmetros encontrados para a isoterma de Freundlich (Tabela 3), o valor de $\mathrm{Kf}$ sugere alta adsorção de cobre e indica grande capacidade do solo em reter o metal. Conforme Sposito (1989) o valor de $n$ relaciona-se qualitativamente à distribuição de sítios energéticos nos coloides do solo. Ainda, segundo Lázaro et al. (2008) valores de $1 / \mathrm{n}$ podem demonstrar se 0 processo de adsorção é favorável (valores entre 0 e 1) ou desfavorável. No caso do solo em estudo, o valor de $1 / \mathrm{n}$ foi de 0,35 , podendo-se considerar que a adsorção do cobre no solo foi favorável.

A modelagem dos dados experimentais com base nas isotermas de Langmuir e Freundlich está expressa na Figura 2.

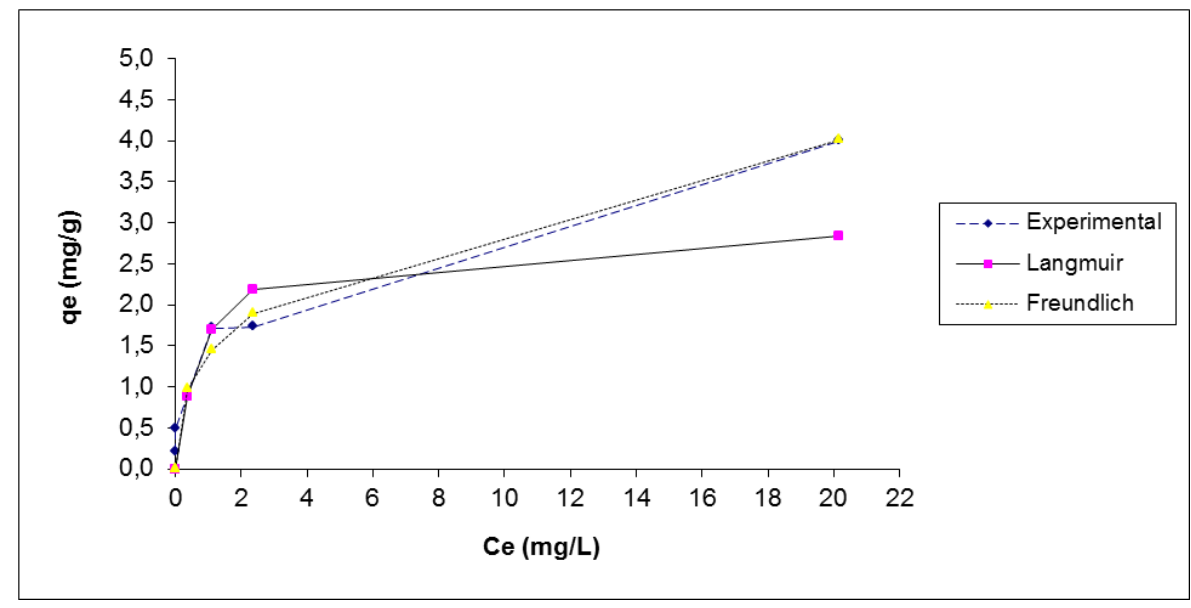

Figura 2 - Isotermas de adsorção do cobre no solo estimadas pelos modelos de Langmuir e Freundlich.

Observa-se claramente que o modelo de Freunclich descreveu melhor o comportamento dos dados experimentais, em relação à adsorção de cobre no solo, confirmando os resultados registrados na Tabela 2, tendo como base os coeficientes de correlação. Nota-se, ainda, que para concentrações mais elevadas de cobre, o modelo de Langmuir subestimou os valores de adsorção do metal pesado no solo.

\section{CONCLUSÕES}

Ao considerar os resultados obtidos nesse trabalho, verifica-se que a capacidade máxima de adsorção de cobre no solo em estudo, determinada pelo modelo de Langmuir, foi de 2,95 mg $\mathrm{g}^{-1}$, e que, apesar de ambos os modelos serem adequados ao estudo de adsorção, a isoterma de Freundlich proporcionou melhor ajuste aos dados experimentais, por ser capaz de descrever a adsorção do cobre no solo.

\section{AGRADECIMENTOS}


Os autores agradecem ao CNPq, à UTFPR e à Fundação Araucária de Apoio ao Desenvolvimento Científico e Tecnológico do Paraná pelo financiamento do projeto.

\section{REFERÊNCIAS BIBLIOGRÁFICAS}

1. AMERICAN PUBLIC HEALTH ASSOCIATION - APHA, AWWA, WEF. Standard methods for the examination of water and wastewater. 20 ed. Washington: American Public Health Association, 1998.

2. ARIAS, M.; PÉREZ-NOVO, C.; LÓPEZ, E.; SOTO, B. Competitive adsorption and desorption of copper and zinc in acid soils. Geoderma, v.133, n.3-4, p.151-159, 2006.

3. BAIRD, C. Química Ambiental. 2 ed. Porto Alegre: Bookman, 2004.

4. BÖTTCHER, J. Use of scaling to quantify variability of heavy matel sorption isotherms. European Journal of Soil Science, v.48, n.2, p.379-386, 1997.

5. EMBRAPA. EMPRESA BRASILEIRA DE PESQUISA AGROPECUÁRIA. Sistema brasileiro de classificação de solos. 2.ed. Rio de Janeiro: Centro Nacional de Pesquisas de Solos, 2006.

6. EMPRESA BRASILEIRA DE PESQUISA AGROPECUÁRIA. Manual de métodos de análise de solo. 2 ed. Rio de Janeiro: Centro Nacional de Pesquisas de Solos, 1997.

7. GARCIA-MIRAGAYA, J.; PAGE, A. L. Sorption of trace quantities of cadmium by soils with different chemical and mineralogical composition. Water, Air and Soil Pollution, v.9, p.289299, 1978.

8. HINZ, C. Description of sorption data with isotherm equations. Geoderma, v.99, p. 225-243, 2001.

9. LÁZARO, D. A.; MANSUR, M. B.; FRANCA, A. S.; OLIVEIRA, L. S.; ROCHA, S. D. F. Performance of cold-pressed cake from Raphanus sativus (L.Var.) oilseeds, a solid residue from biodiesel production, as adsorbent for basic dyes. International Journal of Chemical Engineering, v.1, p.289-302, 2008.

10. LINHARES, L. A.; EGREJA FILHO, F. B.; IANHEZ, R.; SANTOS, E. A. Aplicação dos modelos de Langmuir e Freundlich na adsorção de cádmio e chumbo em diferentes classes de solos brasileiros. Revista Tecnológica, v.17, p.49-60, 2008.

11. LINHARES, L. A.; EGREJA FILHO, F. B.; OLIVEIRA, C. V.; BELLIS, V. M. Adsorção de cádmio e chumbo em solos tropicais altamente intemperizados. Pesquisa Agropecuária Brasileira, v.44, n.3, p.291-299, 2009.

12. NASCIMENTO, C. W. A.; FONTES, R. L. F. Correlação entre características de latossolos e parâmetros de equações de adsorção de cobre e zinco. Revista Brasileira de Ciência do Solo, v.28, p.965-971, 2004.

13. PIVELI, R. P.; KATO, M. T. Qualidade das águas e poluição: aspectos físico-químicos. São Paulo: ABES, 2006.

14. SODRÉ, F. F.; COSTA, A. C. S.; LENZI, E. Adsorção de cobre em solos tropicais com diferentes mineralogias. Acta Scientiarum, v.21, n.3, p.483-489, 1999.

15. SPOSITO, G. The chemistry of soils. New York: Oxford University Press, 1989, 234p. 
16. SOUZA, R. S.; CHAVES, L. H. G.; FERNANDES, J. D. Adsorção de zinco e sua relação com características de solos do Estado da Paraíba. Revista Brasileira de Ciências Agrárias, v.1, p.16, 2006.

17. TEDESCO, M. J.; GIANELLO, C.; BISSANI, C. A.; BOHNEN, H.; VOLKWEISS, S. J. Análises de solo, plantas e outros materiais. 2 ed. Porto Alegre: Departamento de Solos, UFRGS, 1995. 174p.

18. VASCONCELOS, H. L.; LUCHESE, E. B.; LENZI, E. Estudo da fitodisponibilidade do cobre em alguns solos do estado do Paraná. Brazilian Archives of Biology and Technology, v.40, n.3, p.580-585, 1997. 